\title{
EL MERCADO DE LA SEMILLA MEJORADA DE MAÍZ (Zea mays L.) EN MÉXICO. UN ANÁLISIS DEL SALDO COMERCIAL POR ENTIDAD FEDERATIVA
}

\author{
THE CORN (Zea mays L.) IMPROVED SEED MARKET IN MÉXICO. \\ AN ANALYSIS OF THE COMMERCIAL BALANCE BY STATE
}

\author{
J. Alberto García-Salazar y Rocío Ramírez-Jaspeado
}

${ }^{1}$ Economía, Campus Montecillo, Colegio de Postgraduados. Km. 36.5 Carr. México-Texcoco. 56230, Montecillo, Texcoco, Estado de México.

*Autor para correspondencia (jsalazar@colpos.mx)

\section{RESUMEN}

Para proporcionar un panorama sobre la situación del mercado de semilla mejorada de maíz (Zea mays L.) en México se realizó una estimación del consumo de este insumo. El consumo total se comparó con la producción de semilla mejorada para determinar el saldo comercial (déficit o superávit) en cada zona productora de maíz. Se utilizó información de superficie y densidad de siembra por estado, ciclo de producción, régimen hídrico y variedades de maíz, así como importaciones y exportaciones de semilla por aduana y país de origen y destino. Los resultados indican que en el promedio anual del periodo 2008/2010 el consumo total de semilla fue de $\mathbf{1 6 0 . 2}$ miles de toneladas, de las cuales $\mathbf{4 2 . 5} \%$ correspondió a mejorada y el restante a criolla. La producción anual de semilla mejorada fue de 62.5 mil toneladas y el déficit fue mayor a 90 mil toneladas. $\mathrm{El}$ análisis del saldo comercial indica que 26 entidades presentaron un déficit de semilla mejorada, $y$ el mayor ocurrió en los estados de Chiapas, Puebla, Oaxaca, Veracruz y Estado de México, donde superó las 10 mil toneladas en cada entidad. Debido a su importancia en el aumento de la productividad de maíz, se debe promover la producción de semilla mejorada para cubrir el déficit nacional y apoyar a pequeñas empresas productoras de semilla para evitar la existencia de una estructura imperfecta de mercado.

Palabras clave: Zea mays, consumo y producción de semilla, importaciones, MasAgro, saldo comercial.

\section{SUMMARY}

An overview of the market situation of improved maize (Zea mays L.) seeds in México is described here, based on estimation of the consumed quantity for this input. Total consumed quantity was compared with production of improved seed to determine the commercial balance (deficit and surplus) in each corn-producing area. Information collected from every state containing surface production, plant density and yield, as well as production cycles, hydrologic regimes and corn varieties, was used. Results indicate that from 2008 to 2010 the annual average of consumed seed was 160.2 thousand tons, of which $42.5 \%$ was improved seed and the remainder came from local varieties. Annual production of improved seed was 62.5 thousand tons, resulting in a deficit of more than 90 thousand tons. Commercial balance analysis indicates that 26 states have a deficit for improved seed, and the largest deficit occurs in Chiapas, Puebla, Oaxaca, Veracruz and México State, exceeding 10 thousand tons per every state. Since maize productivity depends greatly on the quantity of inputs, improved seed production should be promoted to cover the national deficit, as well as support should be provided to small seed companies to prevent the existence of an imperfect market structure.
Index words: Zea mays, consumption and production of improved seed, imports, MasAgro, commercial balance.

\section{INTRODUCCIÓN}

Un objetivo del Programa de Modernización Sustentable de Agricultura Tradicional (MasAgro) es aumentar la producción de maíz (Zea mays L.) en 13.7 millones de toneladas de grano en las áreas de secano (temporal) de México. El Programa está destinado a pequeños agricultores y se concentra en prácticas agronómicas mejoradas y el uso de semilla de alto rendimiento.

El éxito de MasAgro depende de la adopción, por parte de los agricultores objetivo del programa, de paquetes tecnológicos que utilicen semilla mejorada. Lo anterior significa que se debe aumentar el consumo de semillas mejoradas en México. Para alcanzar esta meta de gran magnitud es necesario plantear interrogantes relacionadas con el consumo del insumo: ¿Cuál es el volumen del consumo de semilla mejorada de maíz y en qué estados se concentra? ¿A cuánto asciende la producción de semilla y cuál es su distribución geográfica? ¿Cuál es el saldo comercial de cada entidad productora de maíz? y ¿En qué estados se debe apoyar la infraestructura productora de semilla mejorada?

Algunas investigaciones y pronunciamientos se han centrado en las interrogantes anteriores. La información del Servicio Nacional de Inspección y Certificación de Semilla (SNICS) señala que la cobertura de la superficie sembrada con semilla certificada de granos básicos (entre ellos el maíz) es aproximadamente 40 \% (Rodríguez-Flores, 2011). La tasa anterior indica un aumento importante en los últimos 15 años, ya que en 1996 sólo $20.3 \%$ de la superficie de maíz se sembraba con variedades mejoradas (Morris y López-Pereira, 2000).

Datos publicados por la Secretaría de Agricultura, Ganadería, Desarrollo Rural, Pesca y Alimentación (SAGARPA, 2010) señalan que en 2010 el consumo de semilla mejorada 
de maíz en el país fue de 162 mil toneladas. En ese año se produjeron 75 mil toneladas con la que se cubrió sólo $47 \%$ de la superficie sembrada total (7.86 millones de hectáreas).

Aunque el uso de semilla mejorada ha aumentado en los últimos 15 años, el padrón de adopción ha sido irregular y su uso se ha concentrado en zonas de producción comercial (Aquino-Mercado et al., 2009). Datos del directorio de productores, obtentores y comercializadores de semilla del 2009 indican que la producción de semilla se concentra en estados del Noroeste y de El Bajío (Luna-Mena et al., 2012). Por ello, no es casualidad que entre los estados con mayor consumo de semilla mejorada se encuentren Sinaloa, Sonora, Jalisco y Guanajuato, en los cuales la superficie sembrada con este tipo de insumo es mayor de 70 \% (OrtegaPaczka, 2003).

Los datos anteriores indican que existe un fuerte déficit de semilla mejorada en México, pero no hay información del saldo comercial (cantidad producida menos cantidad consumida) de semilla mejorada en cada entidad productora. Las razones que justifican efectuar un análisis espacial (geográfico) sobre el déficit o superávit de semilla en cada entidad federativa del país, son las siguientes: a) La semilla mejorada, junto con los fertilizantes y plaguicidas, es un insumo importante para aumentar la productividad de maíz; b) La estimación de la cantidad consumida de semilla mejorada ofrece una idea sobre la magnitud del mercado en cada entidad productora de maíz; c) El cálculo del saldo comercial en el mercado de semilla mejorada permitirá dar una idea sobre el reto tecnológico al que MasAgro se enfrenta, en relación al aumento de la semilla mejorada requerida para cubrir toda la superficie nacional y; d) La identificación de déficits de semilla mejorada en algunas entidades permitirá focalizar apoyos para empresas productoras de semillas que tengan intención de ubicarse en regiones con mercado potencial.

Esta investigación tuvo por objetivo cuantificar la cantidad consumida de semilla mejorada y determinar el saldo comercial a tres niveles, estatal, regional y nacional.

\section{MATERIALES Y MÉTODOS}

\section{Consumo total de semilla y consumo de semilla mejorada de maíz}

La producción agrícola requiere insumos como fertilizantes, mano de obra, plaguicidas y semilla; esta última puede ser criolla o mejorada. Por ser utilizada como insumo, la cantidad consumida de semilla mejorada de maíz está en función de la producción del grano, variable que depende de la superficie cosechada y del rendimiento. Un aumento en la superficie cosechada eleva la producción y aumenta el consumo de insumos como la semilla mejorada.

Es importante mencionar que no se hizo un análisis de demanda que relacione los precios y las cantidades demandadas del producto. De Acuerdo con Nicholson y Snyder (2008), "Una curva de demanda individual muestra la relación entre el precio de un bien y la cantidad de ese bien comprada por un individuo, asumiendo que todos los demás determinantes de la demanda permanecen constantes". Samuelson y Nordhaus (2005) definen a la demanda, o función de demanda, como "la relación entre el precio de mercado de un bien y la cantidad demandada del mismo, si todo lo demás permanece constante." Fisher y Espejo (2004) definen a la demanda como "las cantidades de un producto que los consumidores están dispuestos a comprar a los posibles precios de mercado.” En esta investigación sólo se calculó la cantidad consumida de semilla mejorada.

Tampoco se efectuó un análisis de la demanda derivada que relacione los precios y las cantidades demandadas del insumo, ya que de acuerdo con Parkin et al. (2006) la demanda derivada se puede definir como "la demanda de los distintos factores productivos que se utilizan en el proceso de producción de un bien o servicio". Por su parte, Beattie et al. (2009) señalan que "la demanda para un factor de producción, al igual que la demanda para todos los bienes y servicios, es una relación entre la cantidad del factor usado y los precios"; cuando la producción no cambia se tiene la demanda condicionada de un factor, que de acuerdo con los mismos autores "es definida como la relación entre la cantidad del factor usado y el precio de los factores, manteniendo constante la producción”. Según la FAO (2001), la semilla mejorada, al igual que los fertilizantes y plaguicidas, es una demanda derivada. Dichas definiciones son importantes porque ayudan a diferenciar los diferentes conceptos relacionados con el tema de investigación.

El maíz utilizado como semilla en el proceso de producción es un rubro que se considera en el consumo nacional aparente del grano. El Servicio de Información Agropecuaria y Pesquera (SIAP-SAGARPA, 2011d) reporta el consumo de maíz para semilla, que se suma al consumo de maíz del sector pecuario, de la industria (tortilla, harina, almidones, cereales y alimentos balanceados) y de la población humana. La estimación del consumo de semilla requiere de abundante información sobre superficie y densidad de siembra por estado, ciclos de producción, régimen hídrico y variedades de maíz.

Con $h$ regiones productoras de maíz (y consumidoras de semilla) y $t$ ciclos de producción, el consumo regional (y nacional) de semilla (criolla más mejorada) de maíz blanco $\mathrm{y}$ amarillo se obtiene con las siguientes ecuaciones: 


$$
\begin{gathered}
C S B_{h t}=\operatorname{SSB}_{h t} \times D S_{h} \text { (Ec. 1);CSA } A_{h t}=\operatorname{SSA}_{h t} \times D S_{h} \text { (Ec. 2); } \\
C N S B=\sum_{t=1}^{T} \sum_{h=1}^{H} C S B_{h t}\left(\text { Ec. 3); CNSA }=\sum_{t=1}^{T} \sum_{h=1}^{H} C S A_{h t}(\text { Ec. 4); }\right.
\end{gathered}
$$

donde: $C S B_{h t}=$ consumo de semilla de maíz blanco en la región $h$ en el ciclo de producción $t ; S S B_{h t}=$ superficie sembrada de maíz blanco en la región $h$ en el ciclo de producción $t ; D S_{h}=$ densidad de siembra en la región $h ; C S A_{h t}$ $=$ consumo de semilla de maíz amarillo en la región $h$ en el ciclo de producción $t ; S S A_{h t}=$ superficie sembrada de maíz amarillo en la región $h$ en el ciclo de producción $t ; D S_{h}=$ densidad de siembra en la región $h$; CNSB = consumo nacional anual de semilla de maíz blanco; y $C N S A=$ consumo nacional anual de semilla de maíz amarillo.

La Ecuaciones 1 y 2 se utilizan para calcular el consumo de semilla de maíz blanco y amarillo por región y ciclo de producción; y las Ecuaciones 3 y 4 permiten estimar el consumo nacional anual de semilla (maíz blanco y amarillo).

El consumo total de semilla de maíz blanco y amarillo $\left(C T S_{h}\right)$ en $h$ estaría dado por:

$$
C T S_{h}=\sum_{t=1}^{T} C S B_{h t}+\sum_{t=1}^{T} C S A_{h t}
$$

El consumo de semilla mejorada se calcula usando un método similar:

$$
\begin{gathered}
C S M_{h}=\operatorname{SSM}_{h} \times D S_{h} \\
C N S M=\sum_{h=1}^{H} C S M_{h}
\end{gathered}
$$

donde: $C S M_{h}=$ consumo de semilla mejorada de maíz en la región $h ; S S M_{h}=$ superficie sembrada de maíz con semilla mejorada en la región $h ; D S_{h}=$ densidad de siembra en la región $h$; y CNSM = consumo nacional anual de semilla mejorada de maíz.

La Ecuación 6 sirve para calcular el consumo de semilla mejorada por región, pero no se desagrega por ciclos de producción debido a la ausencia de información sobre superficie sembrada en los ciclos primavera-verano y otoño invierno. La Ecuación 7 se emplea para calcular el consumo nacional anual de semilla mejorada.

En este punto es importante definir que el consumo de semilla mejorada (estimado con la Ecuación 6) es igual a la cantidad real consumida, en tanto que el consumo total de semilla (estimada con la Ecuación 5) es igual a la cantidad potencial consumida, es decir, la que existiría en caso de que la totalidad de la superficie de maíz fuera sembrada con semilla mejorada. Es conveniente mencionar que habría sido importante hacer una mayor desagregación de los datos de consumo de semilla; por ejemplo, diferenciar entre semillas de maíz híbrido y semillas de polinización abierta; sin embargo, la inexistencia de información sobre superficie sembrada con ambos tipos de semilla a nivel regional imposibilitó esta estimación.

\section{Déficit o superávit de semilla a través del saldo comercial}

Cuando la cantidad ofrecida sobrepasa a la consumida se genera un superávit, también llamado excedente; cuando la producción es inferior a la cantidad consumida se genera un déficit (Mochón, 2006).

En este punto también es importante recalcar que no se hizo un análisis de oferta que relacione los precios y las cantidades ofrecidas. Nicholson y Snyder (2008) indican que "la curva de oferta de corto plazo de la empresa muestra qué tanto va a producir ésta a varios posibles precios del producto". Fischer y Espejo (2004) mencionan que "la oferta se refiere a las cantidades de un producto que los productores están dispuestos a producir a los posibles precios del mercado". Parkin et al. (2006) indican que "el término oferta se refiera a la relación completa entre el precio de un bien y la cantidad ofrecida del mismo". En esta investigación se consideró la producción de semilla mejorada.

El saldo comercial de semilla mejorada se obtuvo restando a la producción de semilla mejorada el consumo total de semilla:

$$
S C_{h}=P S M_{h}-C T S_{h}
$$

donde: $S C_{h}=$ saldo comercial de semilla en la región $h$; $P S M_{h}=$ producción de semilla mejorada en la región $h ; \mathrm{y}$ $\operatorname{CTS}_{h}=$ consumo total de semilla en la región $h$.

Un saldo positivo indica un superávit de semilla mejorada, que será enviado a regiones con producción insuficiente. Un saldo negativo muestra la existencia de un déficit por semilla mejorada que puede ser abastecido con semilla proveniente de estados excedentarios o mediante importaciones. En ausencia de semilla mejorada se usa la semilla criolla.

A continuación se indican las fuentes de información respectivas de los datos usados para estimar el consumo de semilla: (1) Superficie sembrada por estado, régimen hídrico y tipo de variedad, que se obtuvieron del Servicio de Información Agropecuaria y Pesquera de la Secretaría de Agricultura, Ganadería, Desarrollo Rural, Pesca y Alimentación (SIAP-SAGARPA, 2011a); (2) Densidad de siembra 
por régimen hídrico, que se obtuvo de los cuadros de costos de producción que reportó SIAP-SAGARPA (2011c); (3) Importaciones y exportaciones de semilla de maíz, que se obtuvieron del sistema de información comercial de México (SIAP-SAGARPA, 2011b); (4) Superficie sembrada con semilla mejorada, según estadísticas del Instituto Nacional de Estadística y Geografía (INEGI, 2008-2010); y (5) Producción de semilla mejorada por entidad federativa, que se obtuvo del Servicio Nacional de Inspección y Certificación de Semilla (SNICS, 2011).

\section{RESULTADOS Y DISCUSIÓN}

\section{Consumo total de semilla y consumo de semilla mejorada de maíz}

Con base en la densidad de siembra promedio que se aplica en tecnologías de riego y temporal, y la superficie sembrada en diferentes regímenes hídricos y ciclos de producción, se estimó el consumo de semilla (Cuadro 1), el cual fue de 160 mil toneladas: $95 \%$ correspondió a maíz blanco y $5 \%$ a maíz amarillo. El mayor consumo de semilla mejorada se presentó en las regiones Sur y Centro con más de 33 mil toneladas en cada una, seguidas de la región Occidente que participó con 19.7 \% de la cantidad consumida total.

El consumo de semilla de maíz blanco se concentró en $\mathrm{Si}$ naloa, Chiapas, Oaxaca, Puebla, Veracruz, Edo. de México y Jalisco, con 8.2, 8.0, 7.9, 7.9, 7.6, 7.3 y 7.2 \% del consumo total. El consumo de semilla de maíz amarillo se concentró en Chihuahua, Chiapas y Tamaulipas, con 29.1, 21.4 y 16.5 $\%$ del consumo total (Cuadro 1).

Los Estados con mayor consumo fueron Chiapas, Sinaloa, Jalisco, Puebla, Oaxaca, Veracruz, y Edo. de México, con cantidades que oscilaron entre 10 y 14 mil toneladas de semilla. En Guerrero, Michoacán, Guanajuato, Zacatecas, San Luis Potosí e Hidalgo la cantidad consumida fue mayor a 5 mil pero menor a 10 mil toneladas. El consumo de semilla fluctuó entre 1 y 5 mil toneladas en Chihuahua, Durango, Tamaulipas, Yucatán, Campeche, Querétaro, Tlaxcala, Tabasco y Quintana Roo. Las demás entidades presentaron un consumo menor a mil toneladas.

La cantidad consumida del insumo se calculó con base en la superficie sembrada con semilla mejorada y la densidad de siembra, ascendió a 68 mil toneladas (Cuadro 1), cifra que representa $42.5 \%$ del consumo total; las restantes 92 mil toneladas corresponden a semilla criolla. Las cifras anteriores indican que más de la mitad de la superficie sembrada con maíz no utiliza semilla mejorada, y que se tiene una amplia brecha para elevar el potencial productivo del grano.
En cuanto a la distribución del consumo de semilla mejorada por entidad federativa (Cuadro 1), sobresalen Jalisco y Sinaloa con más de 10 mil toneladas. En Michoacán, Guanajuato, Edo. de México y Guerrero la cantidad consumida de semilla mejorada se ubicó entre 4 y 6 mil toneladas; en Chiapas, Veracruz, Zacatecas, Tamaulipas y Chihuahua, el consumo se ubicó entre 2 y 4 mil toneladas; y en el resto de las entidades el consumo fue inferior a 2 mil toneladas.

En más de la mitad de la superficie sembrada con maíz se utilizó semilla criolla (Cuadro 1). En Chiapas, Oaxaca y Puebla la cantidad consumida de semilla criolla superó las 10 mil toneladas; en Veracruz fue mayor a 8 mil toneladas; en el Edo. de México, Guerrero, San Luis Potosí e Hidalgo el consumo de semilla criolla varió entre 4 y 8 mil toneladas y en las demás entidades fue inferior a 4 mil toneladas.

En el promedio anual del periodo 2009/2010, la producción de semilla mejorada de maíz fue 63 mil toneladas. Esta producción está concentrada en el Noroeste y Occidente, regiones que producen 48.4 y $39.5 \%$ de la producción nacional. Existen regiones, como la Península y el Golfo donde apenas se producen 27 y 295 toneladas de semilla mejorada (Cuadro 1). Además, $36.4 \%$ de la producción de semilla se ubica en Sinaloa, $26.14 \%$ en Guanajuato, $10.9 \%$ en Jalisco y $9.2 \%$ en Nayarit; estas cuatro entidades concentraron $82.9 \%$ de la producción. El resto de la producción de semilla mejorada está dispersa en el territorio nacional (Cuadro 1).

\section{Déficit o superávit de semilla a través del saldo comercial}

Sólo el Noroeste presenta un superávit de semilla mejorada por 15.8 mil toneladas. Las demás regiones presentan déficit, el cual es mayor en el Sur y Centro del país por más de 30 mil toneladas en cada una.

El superávit total de semilla mejorada a nivel nacional es de 24.1 mil toneladas, y el déficit total de 122.0 mil toneladas, y al sumar ambos saldos se tiene un déficit total de semilla mejorada por 97.8 mil toneladas (Cuadro 1). Los estados con superávit de semilla mejorada se encuentran en entidades del Noroeste y Occidente del país, como Sinaloa con más de 10 mil toneladas, Guanajuato mayor a 8 mil toneladas, y Nayarit con casi 5 mil toneladas. Sonora, Baja California Sur y Colima presentan superávits pequeños, menores a $1 \mathrm{mil}$ toneladas.

La mayor parte del territorio nacional presenta déficits de semilla mejorada y los más altos se presentan en Chiapas, Puebla, Oaxaca, Veracruz y Edo. de México, donde superan las 10 mil toneladas. En Guerrero, Michoacán, Jalisco, San Luis Potosí, e Hidalgo, los déficits se ubicaron entre 5 y $10 \mathrm{mil}$ toneladas (Cuadro 1). 
Cuadro 1. Consumo, producción y saldo anual de semilla de maíz por región y Estado, en promedio del periodo 2008/2010. Cifras en toneladas.

\begin{tabular}{|c|c|c|c|c|c|c|c|}
\hline \multirow{2}{*}{ Estado } & \multicolumn{5}{|c|}{ Consumo de semilla de maíz } & \multirow{2}{*}{ Producción $^{\dagger}$} & \multirow{2}{*}{ Saldo } \\
\hline & Blanco & Amarillo & Total & Mejorada & Criolla & & \\
\hline \multicolumn{8}{|l|}{ Región } \\
\hline Noroeste & 14,103 & 306 & 14,409 & 11,763 & 2645 & 30,283 & $+15,874$ \\
\hline Norte & 17,995 & 2519 & 20,514 & 6990 & 13,524 & 1947 & $-18,567$ \\
\hline Noreste & 2640 & 1332 & 3972 & 2545 & 1428 & 1756 & -2216 \\
\hline Occidente & 30,004 & 1295 & 31,299 & 21,859 & 9440 & 24,730 & -6569 \\
\hline Centro & 33,540 & 369 & 33,909 & 9042 & 24,867 & 2259 & $-31,650$ \\
\hline Sur & 33,897 & 1727 & 35,623 & 9327 & 26,296 & 1248 & $-34,375$ \\
\hline Golfo & 13,165 & 166 & 13,331 & 3890 & 9442 & 295 & $-13,036$ \\
\hline Península & 6834 & 334 & 7168 & 2756 & 4412 & 27 & -7140 \\
\hline Total & 152,178 & 8048 & 160,226 & 68,172 & 92,054 & 62,546 & $-97,679$ \\
\hline \multicolumn{8}{|c|}{ Entidad federativa } \\
\hline Aguascalientes & 999 & 0 & 999 & 199 & 800 & 15 & -984 \\
\hline Baja C. Sur & 84 & 0 & 84 & 70 & 14 & 230 & +146 \\
\hline Campeche & 2755 & 0 & 2755 & 1889 & 866 & 27 & -2728 \\
\hline Chiapas & 12,189 & 1720 & 13,910 & 3559 & 10,350 & 992 & $-12,918$ \\
\hline Chihuahua & 2574 & 2342 & 4917 & 2379 & 2537 & 211 & -4705 \\
\hline Coahuila & 674 & 11 & 685 & 127 & 558 & 27 & -659 \\
\hline Colima & 270 & 0 & 271 & 214 & 57 & 364 & +93 \\
\hline D. F. & 115 & 0 & 115 & 2 & 113 & 0 & -115 \\
\hline Durango & 3834 & 0 & 3834 & 1302 & 2533 & 14 & -3821 \\
\hline Guanajuato & 8130 & 36 & 8166 & 4883 & 3282 & 16,323 & +8157 \\
\hline Guerrero & 9690 & 6 & 9697 & 4160 & 5537 & 193 & -9504 \\
\hline Hidalgo & 5168 & 32 & 5200 & 985 & 4215 & 110 & -5091 \\
\hline Jalisco & 10,979 & 1244 & 12,223 & 10,928 & 1295 & 6809 & -5414 \\
\hline México & 11,159 & 133 & 11,292 & 4162 & 7130 & 335 & $-10,957$ \\
\hline Michoacán & 9626 & 15 & 9641 & 5635 & 4005 & 1219 & -8422 \\
\hline Morelos & 494 & 21 & 515 & 461 & 54 & 174 & -341 \\
\hline Nayarit & 869 & 45 & 914 & 632 & 282 & 5760 & +4846 \\
\hline Nuevo León & 499 & 2 & 501 & 108 & 392 & 0 & -501 \\
\hline Oaxaca & 12,017 & 0 & 12,017 & 1,608 & 10,409 & 64 & $-11,953$ \\
\hline Puebla & 11,979 & 93 & 12,072 & 1,742 & 10,330 & 64 & $-12,008$ \\
\hline Querétaro & 2392 & 5 & 2397 & 765 & 1632 & 1505 & -892 \\
\hline Quintana Roo & 1359 & 10 & 1369 & 333 & 1036 & 0 & -1369 \\
\hline San Luis Potosí & 5341 & 0 & 5341 & 633 & 4707 & 0 & -5341 \\
\hline Sinaloa & 12,423 & 261 & 12,684 & 10,494 & 2190 & 22,941 & $+10,257$ \\
\hline Sonora & 727 & 0 & 727 & 568 & 159 & 1351 & +624 \\
\hline Tabasco & 1660 & 0 & 1660 & 776 & 884 & 0 & -1660 \\
\hline Tamaulipas & 2142 & 1330 & 3472 & 2436 & 1035 & 1756 & -1715 \\
\hline Tlaxcala & 2233 & 86 & 2319 & 926 & 1393 & 72 & -2247 \\
\hline Veracruz & 11,505 & 166 & 11,672 & 3114 & 8558 & 295 & $-11,376$ \\
\hline Yucatán & 2720 & 323 & 3044 & 534 & 2509 & 0 & -3044 \\
\hline Zacatecas & 5572 & 165 & 5737 & 2548 & 3189 & 1696 & -4042 \\
\hline Total & 152,178 & 8048 & 160,226 & 68,172 & 92,054 & 62,546 & $-97,679$ \\
\hline
\end{tabular}


Por el lado de la oferta el mercado de semilla mejorada de maíz está concentrado en algunas empresas. Datos del SNICS (2011) indican que 85.3 \% de la producción es generada por grandes empresas, lo cual indica que existe una estructura de mercado imperfecta. Apenas $9.1 \%$ de la producción está en manos de empresas pequeñas, $3.8 \%$ proviene de las sociedades de producción rural, y $1.9 \%$ es producido por personas físicas e instituciones de investigación (Cuadro 2).

La existencia de una estructura de competencia imperfecta en el mercado de la semilla mejorada no es deseable para el consumidor (productor de maíz), por las siguientes razones: 1) El reducido número de empresas permite que éstas tengan el poder de establecer el precio, y 2) El productor acepta la calidad de semillas que ofrecen esas empresas. Tampoco es deseable para la sociedad que exista poca competencia en el mercado de semillas mejoradas porque los altos precios ocasionarán un bajo consumo del insumo, y por ende menor rendimiento.

El monopolio en el comercio de las semillas ha propiciado que los precios de venta de las semillas híbridas en México sean los más altos del mundo. Mil semillas de maíz se cotizan en 2.7 dólares, en comparación con 1.3 dólares en la faja maicera de Estados Unidos (Espinosa-Calderón et al., 2010).

Los datos de comercio exterior de semilla de maíz indican que México realiza importaciones y exportaciones de manera considerable. En promedio del periodo 2008/2010, las importaciones anuales de semilla de maíz amarillo fueron de 9.6 miles de toneladas; las exportaciones de semilla fueron en su mayoría de maíz blanco y ascendieron a 7.6 mil toneladas (Cuadro 3). Las importaciones se realizaron por fronteras del norte y puertos ubicados en el Golfo de México: $28.1 \%$ se internaron por Tuxpan, $25.1 \%$ por Nogales, $14.9 \%$ por Piedras Negras y $12.5 \%$ por Mexicali. El país de procedencia de las importaciones de semilla fueron los EE. UU., país que concentró $64.8 \%$, seguido por Brasil de dónde provino $21.1 \%$ de la compras de semilla.

Por el volumen de las importaciones de semilla de maíz amarillo se deduce que éstas abastecen el consumo en este mercado, lo que significa que la producción de semilla mejorada de maíz amarillo no existe o es marginal. Al respecto, no es recomendable la dependencia del mercado externo para abastecer el consumo de semilla de maíz amarillo, porque los costos de transporte del país de origen hasta el lugar de consumo elevan el precio de la semilla, y se tiene que aceptar la calidad de semilla proveniente del extranjero.

Las exportaciones de semilla se realizaron por puertos del Pacífico y Golfo de México, y por la frontera norte: 31.4 \% salieron por Manzanillo, Colima; 30.7 \% por Altamira, Tamaulipas; $12.9 \%$ por Ciudad Hidalgo, Chiapas, y $12.5 \%$ por Palomas, Chihuahua. El destino de las exportaciones de semilla fueron Venezuela que adquirió $54.8 \%$ de estas exportaciones, seguido de EE. UU. y Guatemala que importaron 23.4 y $6.5 \%$ de las ventas de semilla de maíz realizadas por México (Cuadro 3).

Con la Ley de Producción, Certificación y Comercio de Semillas (LPCCS), en 1961 se dio un fuerte impulso a la industria productora de semilla donde el mejoramiento genético estaba a cargo del gobierno para atender las funciones específicas del sistema agrícola que el país requería, restringiendo así la participación de la industria privada.

En la década de los noventas, las políticas de globalización comercial obligaron a que el gobierno mexicano realizara una serie de cambios en su legislación; la nueva LPCCS permitió la participación sin restricciones del sector privado en la investigación, producción y comercialización de semilla; las empresas transnacionales invadieron rápidamente el mercado y redujeron el crecimiento de las empresas nacionales ya existentes y restringieron el surgimiento de las nuevas (Luna-Mena et al., 2012). En la actualidad la

Cuadro 2. Producción anual de semilla mejorada de maíz por tipo de productor, en promedio del periodo 2009/2010. Cifras en toneladas.

\begin{tabular}{|c|c|c|c|c|}
\hline Productor & 2009 & 2010 & Promedio & $\%$ \\
\hline Empresas grandes & 54,749 & 51,909 & 53,329 & 85.3 \\
\hline Empresas pequeñas & 6348 & 5049 & 5698 & 9.1 \\
\hline Instituciones de investigación & 298 & 342 & 320 & 0.5 \\
\hline Sociedades de producción rural & 1795 & 2904 & 2350 & 3.8 \\
\hline Personas físicas & 984 & 719 & 852 & 1.4 \\
\hline Total & 64,174 & 60,924 & 62,549 & 100.0 \\
\hline
\end{tabular}

Fuente Elaboración propia con datos del SNICS (2011). 
Cuadro 3. Importaciones y exportaciones anuales de semilla por aduana y país, en promedio del periodo 2008/2010. Cifras en toneladas.

\begin{tabular}{lrrlrr}
\hline \multirow{2}{*}{ Aduana/país } & \multicolumn{2}{c}{ Importaciones } & & \multicolumn{2}{c}{ Exportaciones } \\
\cline { 2 - 5 } Nuevo Laredo & \multicolumn{1}{c}{ Volumen } & $\%$ & Aduana/país & Volumen & $\%$ \\
Matamoros & 9 & 0.1 & Guadalajara & 153 & 2.0 \\
P. Negras & 83 & 0.9 & Altamira & 2330 & 30.7 \\
Cd. Juárez & 1429 & 14.9 & Puerto Vallarta & 158 & 2.1 \\
Nogales & 3 & 0 & Nvo. Amanecer & 124 & 1.6 \\
Mexicali & 2405 & 25.1 & Nogales & 12 & 0.2 \\
Tuxpan & 1204 & 12.5 & Nuevo Laredo & 182 & 2.4 \\
Veracruz & 2693 & 28.1 & Veracruz & 39 & 0.5 \\
Coatzacoalcos & 467 & 4.9 & Ciudad Hidalgo & 981 & 12.9 \\
Progreso & 230 & 2.4 & Palomas & 947 & 12.5 \\
Nvo. Progreso & 144 & 1.5 & Manzanillo & 2385 & 31.4 \\
Reynosa & 520 & 5.4 & Colombia & 65 & 0.9 \\
Total & 413 & 4.3 & Reynosa & 217 & 2.9 \\
& 9599 & 100 & Total & 7595 & 100.0 \\
EE. UU. & & & & 5161 & 54.8 \\
Brasil & 6151 & 64.8 & Venezuela & 1777 & 23.4 \\
Guatemala & 2000 & 21.1 & Estados Unidos & 481 & 6.3 \\
Colombia & 436 & 4.6 & Guatemala & 426 & 5.6 \\
Argentina & 350 & 3.7 & El Salvador & 330 & 4.3 \\
Chile & 361 & 3.8 & Colombia & 202 & 2.7 \\
Otros & 55 & 0.6 & Perú & 217 & 2.9 \\
Total & 146 & 1.5 & Otros & 7594 & 100.0 \\
\hline
\end{tabular}

Fuente: Elaboración propia con información obtenida de SIAP-SAGARPA (2011b).

industria de semilla en México se compone de empresas multinacionales, de empresas privadas nacionales, agricultores individuales y dependencias de investigación (USDAFAS, 2008).

Los datos anteriores indican un bajo consumo de semilla mejorada en relación con la cantidad potencial consumida. ¿Cómo pues aumentar el consumo de semilla mejorada? La respuesta a esta interrogante hace necesario analizar los factores que determinan la cantidad consumida de semilla mejorada de maíz. Desde el punto de vista económico, los factores más importantes que afectan la cantidad consumida del insumo son: el precio de la semilla, el ingreso del productor (consumidor de maíz), el precio de los bienes complementarios en el proceso de producción (fertilizantes, mano de obra, etc.), y la superficie sembrada.

Evidencia empírica reciente indica que la superficie sembrada, el tamaño del predio, la productividad, el nivel so- cioeconómico y la rentabilidad de los productores, son algunos de los factores que explican el potencial de mercado de semilla mejorada de maíz (Donnet et al., 2012).

La ley de la demanda establece que un menor precio de la semilla mejorada determinará una mayor cantidad consumida, por tanto es indispensable que el precio de la semilla mejorada sea lo más bajo posible. Como el precio de la semilla que el productor paga considera el costo de transporte de enviar el insumo desde la planta de producción hasta la zona de consumo (parcela), es indispensable que éste sea bajo. Por ello, el establecimiento de empresas productoras de semilla certificada en forma local y regional dirigidas a nichos de mercado específicos, como productores de pequeña escala en lugares donde carecen de este tipo de insumos, permitiría un mejor abastecimiento (Luna-Mena et al., 2012) y reduciría el precio del insumo pues se eliminarían costos de movilización de la semilla desde otras regiones. 
Por tanto, MasAgro debe promover el establecimiento de empresas productoras de semilla mejorada en las entidades donde se localiza el consumo, o lo más cercanas posible, pues el uso de semilla mejorada es mayor/menor en las entidades cercanas/alejadas de los centros de producción. Es importante formular un programa especial de abastecimiento de semilla mejorada que asegure el abasto en tiempo y forma del insumo.

Algunas investigaciones (Córdova et al., 2002) señalan que los agricultores ubicados en áreas de producción aisladas en laderas con suelos pobres, generalmente no tienen acceso a la semilla proveniente de los sistemas convencionales de producción de semilla, ya sea porque no son sujetos de crédito o porque la industria de semillas no se interesa en esas áreas, pues sus ganancias no son atractivas. Este problema se ha solucionado a través del desarrollo de sistemas no convencionales de acondicionamiento de semillas. En consecuencia, debe ser objetivo de MasAgro hacer llegar la semilla mejorada a esas regiones alejadas de las vías de comunicación. Al respecto, en Honduras se han realizado proyectos para la producción de semillas mejoradas de granos básicos orientados a pequeños agricultores, con el objetivo de incrementar la producción y la productividad a través del uso sostenible de semillas de buena calidad (FAO, 2011)

Otro factor que afecta la cantidad consumida es el ingreso de productor. El bajo nivel de utilización de semilla mejorada, menor a $50 \%$, indica que las necesidades del productor de maíz no están saturadas en relación el consumo de este insumo; por tanto, la semilla mejorada es un bien normal, lo cual indica que hay una relación positiva entre la cantidad consumida de semilla mejorada y el ingreso del productor: aumentos en el ingreso tendrán como efecto un aumento en la cantidad consumida. El hecho de que un incremento del ingreso del productor por unidad de superficie pueda venir de un aumento en el precio del maíz, o bien por un mayor rendimiento por hectárea, crean un círculo entre el rendimiento y el consumo de semilla mejorada. Para elevar la cantidad consumida de semilla es necesario aumentar el ingreso del productor, vía mayor rendimiento; y para aumentar éste es necesario elevar la demanda de semilla mejorada.

El problema anterior se resuelve aumentando el precio del maíz, variable determinada por el mercado. Sobre este punto es importante mencionar que proyectos de acopio y comercialización de empresas comercializadoras que incluyan programas de inventarios serían una vía efectiva para evitar la caída estacional de los precios. Los productores deberán almacenar parte de la cosecha en los meses de alta producción y sacar sus inventarios en las temporadas de producción escasa. Un programa de tal naturaleza ayuda a estabilizar los precios que recibe el productor (GarcíaSalazar et al., 2000). Medidas como la anterior requieren de infraestructura de almacenamiento para la implementación de programas de inventarios. Por tanto, MasAgro deberá complementarse con políticas que promuevan el establecimiento de almacenes en aquellas regiones donde dicha infraestructura sea insuficiente.

Políticas encaminadas al apoyo del productor, como PROCAMPO, permitirán también elevar el nivel de ingreso del productor, y por tanto, se elevaría también el uso de semilla mejorada. En resumen, el aumento del consumo de semilla mejorada requiere de la permanencia de políticas de subsidios que apoyen el ingreso del productor.

El precio de los insumos usados como complemento en el proceso de producción es otro factor que afecta la cantidad consumida de maíz. El uso de semilla mejorada está relacionado con la aplicación de paquetes tecnológicos modernos donde la semilla se complementa con otros insumos, como los fertilizantes. Una disminución en el precio del fertilizante ocasionará un aumento en la cantidad consumida de este insumo y en el uso de semilla mejorada. Lo anterior plantea que MasAgro debe considerar políticas que promuevan el aumento de la competitividad de la industria de fertilizantes de maíz en México. Algunos estudios han determinado que el incremento en la productividad del maíz depende de la reducción de los precios de los fertilizantes (Salgado-Vega y Miranda-González, 2010).

Otro factor que afecta la cantidad consumida de semilla mejorada es el tamaño del predio. A mayor tamaño de predio, el ingreso obtenido en la unidad de producción también será mayor y aumentará la posibilidad de destinar un mayor porcentaje del ingreso a la adquisición de insumos mejorados como la semilla. En México, la cantidad consumida de semilla mejorada está en función directa de la superficie sembrada con este tipo de semilla. Por ejemplo, los agricultores orientados a la producción comercial que disponen de grandes extensiones de tierra tienen como proveedores de semilla a las principales comercializadoras: Monsanto, Pioneer y Syngenta (Luna-Mena et al., 2012), lo que les garantiza un mayor rendimiento y por ende un mayor ingreso.

\section{CONCLUSIONES}

En el periodo 2008/2010 el consumo anual de semilla mejorada de maíz en México representó $42.7 \%$ del consumo total de semilla, que ascendió a 160 mil toneladas. La producción de semilla mejorada fue de 63 mil toneladas y se concentró en empresas grandes. Al comparar la producción de semilla mejorada con la cantidad consumida total se encuentra un saldo comercial negativo por más de $95 \mathrm{mil}$ 
toneladas. De las ocho regiones consideradas en la investigación, solo el Noroeste presentó superávits de semilla y el resto déficits, principalmente en el Centro y Sur del país. En la mayor parte de las entidades federativas del país la cantidad consumida de semilla fue superior a la oferta y los mayores déficits se ubicaron en Chiapas, Puebla, Oaxaca, Veracruz y Estado de México. La diferencia entre el consumo y la producción de semilla mejorada fue abastecida con importaciones provenientes, principalmente, de los Estados Unidos y se usaron para la producción de maíz amarillo.

Debido a la fuerte concentración del consumo en el Noroeste del país, es recomendable desconcentrar la producción del insumo y estimular la oferta en las regiones con mayor consumo potencial ubicadas en las entidades deficitarias, principalmente en el Centro y Sur del país. Además, es importante promover la producción de semilla mejorada en empresas pequeñas que garanticen la competencia en la distribución del insumo. Por el fuerte déficit nacional y la existencia de importaciones, que acentúan la dependencia hacia el exterior en materia de maíz, se recomienda fomentar la participación del Gobierno mediante programas como MasAgro que permitan incentivar e incrementar la producción de semilla mejorada.

\section{AGRADECIMIENTO}

La presente investigación fue financiada con recursos del Programa de Modernización Sustentable de Agricultura Tradicional (MasAgro) dirigido por el Centro Internacional de Mejoramiento de Maíz y Trigo (CIMMYT) y la Secretaría de Agricultura, Ganadería, Desarrollo Rural, Pesca y Alimentación (SAGARPA).

\section{LITERATURA CITADA}

Aquino-Mercado P., R. J. Peña y I. Ortiz-Monasterio (2009) México y el CIMMYT Disponible en: http://www.cimmyt.org/en/aboutus/partnerships/countries/doc_view/668-mexico-y-el-cimmyt (Junio 2012).

Beattie B. R., C. R. Taylor and M. J. Watts (2009) The Economics of Production. 2nd ed. Krieger Pub. Co. Malabar, Florida, USA. 299 p.

Córdova H., S. Castellanos, H. Barreto y J. Bolaños (2002) Veinticinco años de mejoramiento en los sistemas de maíz en Centroamérica: logros y estrategias hacia el año 2000. Agronomía Mesoamericana 13:73-84.

Donnet L., D. López, J. Arista, F. Carrión, V. Hernández y A. González (2012) El potencial de mercado de semillas mejoradas de maíz en México. Programa de Socioeconomía, Centro Internacional de Mejoramiento de Maíz y Trigo (CIMMYT), Documento de Trabajo 8. México. 21 p.

Espinosa-Calderón A., M. Tadeo-Robledo y A. Turrent- Fernández (2010) Concentración de la oferta de semillas mejoradas de maíz. Disponible en: http://www.jornada.unam.mx /2010/03/13/oferta.html (Junio 2012).

FAO, Food and Agriculture Organization of the United Nations (2001) Reform and decentralization of agricultural services a policy framework. Disponible en: http://www.fao.org/docrep/005/ Y2006E/Y2006E00.HTM (Agosto 20013).

FAO, Organización de las Naciones Unidas para la Agricultura y la
Alimentación (2011) Proyecto de Reforzamiento de las Polí ticas de Semilla de Granos Básicos en Apoyo a la Agricultura Campesina. Desgranadoras Manuales: Una Buena Práctica en la Producción y Beneficiado en la Semilla de Maíz. Disponible en: http://www.pesacentroamerica.org/semillas/guia_desgranadoras_honduras.pdf (Junio, 2012).

Fisher L. y J. Espejo (2004) Mercadotecnia. 3a ed. Ed. McGraw Hill. México. $264 \mathrm{p}$.

García-Salazar J. A., J. A. Matus-Gardea, M. A. Martínez-Damián, M. J. Santiago-Cruz y A. Martínez-Garza (2000) Determinación de la demanda óptima de almacenamiento de maíz en México. Agrociencia 34:773-784.

INEGI, Instituto Nacional de Estadística y Geografía (2008-2010) Anuario Estadístico del Estado. Disponible en: http://www.inegi.org.mx/prod_serv/contenidos/espanol/biblioteca/Default. asp? accion $=15 \& u p c=702825201760$ (Junio 2012).

Luna-Mena B. M., M. A. Hinojosa-Rodríguez, O. J. Ayala-Garay, F. Castillo-González y J. A. Mejía-Contreras (2012) Perspectivas de desarrollo de la industria semillera de maíz en México. Revista Fitotecnia Mexicana 35:1-7.

Mochón F. (2006) Principios de Economía. 3a ed. Ed. McGraw Hill. 370 p.

Morris M. L. y M. A. López-Pereira (2000) Impactos del Mejoramiento de Maíz en América Latina, 1966-1997. Disponible en: http:// apps.cimmyt.org/research/Economics/ map/impact_studies/ ImpactsMaize66_97/ImpactosLA/pdfs/ImpactosLA_agrad. pdf (Junio 2012).

Nicholson W. and Ch. Snyder (2008) Microeconomic Theory, Basic Principles and Extensions. 10th ed. South-Western CENGAGE Learning. Mason OH, USA. 740 p.

Ortega-Paczka R. (2003) El maíz como cultivo II: In: Sin Maíz no hay País. José Vicente Anaya (ed). CONACULTA. pp:123-154.

Parkin M. y G. Esquivel, M. Ávalos (2006) Microeconomía, Versión para América Latina. 7a ed. Ed. Pearson Educación. México. 584 p.

Rodríguez-Flores I. (2011) Semillas certificadas, origen de la competitividad. 2000 AGRO Revista Industrial del Campo 69:1-12.

SAGARPA, Secretaría de Agricultura, Ganadería, Desarrollo Rural, Pesca y Alimentación (2010) Cuarto Informe de Labores de SAGARPA. México, D.F. 178 p.

Salgado-Vega M. C. y .S Miranda-González (2010) Panorama de la agricultura en México para 2010. Economía Actual 3:10-12.

Samuelson P. A. y W. D Nordhaus (2005) Economía. 18a ed. Ed. McGraw-Hill Interamericana. México. $810 \mathrm{p}$.

SIAP-SAGARPA, Servicio de Información Agroalimentaria y Pesquera y Secretaría de Agricultura, Ganadería, Desarrollo Rural, Pesca y Alimentación (2011a) Información básica, Agricultura, Producción anual. Disponible en: www.siap.sagarpa.gob. $\mathrm{mx} /$ index.php?option $=$ com_content\&view $=$ article\&id $=10 \& \mathrm{It}$ emid $=15$ (Octubre 2011).

SIAP-SAGARPA, Servicio de Información Agroalimentaria y Pesquera y Secretaría de Agricultura, Ganadería, Desarrollo Rural, Pesca y Alimentación (2011b) Información básica, Economía y mercados. Seguimiento oportuno del comercio exterior. Disponible en: www.siap.sagarpa.gob.mx/index.php?option=com content\&view $=$ article\&id=50\&Itemid=380 (Octubre 2011).

SIAP-SAGARPA, Servicio de Información Agroalimentaria y Pesquera de la Secretaría de Agricultura, Ganadería, Desarrollo Rural, Pesca y Alimentación (2011c) Sistema Producto, Maíz, Producción, Costos. Disponible en: http://www.campomexicano. gob.mx (Diciembre 2011).

SIAP-SAGARPA, Servicio de Información Agroalimentaria y Pesquera) Secretaría de Agricultura, Ganadería, Desarrollo Rural, Pesca y Alimentación (2011d) Información Básica, Agricultura, Balanza Nacional Disponibilidad-Consumo. Disponible en: www.siap.sagarpa.gob.mx/index.php?option=com_content \&view=article\&id=12\&Itemid=17. (Octubre 2011).

SNICS, Servicio Nacional de Inspección y Certificación de Semilla (2011) Semilla de Maíz 2009/2010. Información proporcionada en CD en las oficinas centrales del SNICS. Tlalnepantla, Estado de México, Noviembre de 2011.

USDA-FAS, United States Department of Agriculture, Foreign Agricultural Service (2008) Mexico Planting Seeds Annual 2008. Disponible en: http://www.fas.usda.gov/gainfiles/200808/146295565.pdf (Mayo 2013). 